\title{
Formation of Fermi surfaces and the appearance of liquid phases in holographic theories with hyperscaling violation
}

\author{
Xiao-Mei Kuang, ${ }^{a}$ Eleftherios Papantonopoulos, ${ }^{a}$ Bin Wang ${ }^{b}$ and Jian-Pin $\mathbf{W u}{ }^{c, d}$ \\ ${ }^{a}$ Department of Physics, National Technical University of Athens, \\ GR-15780 Athens, Greece \\ ${ }^{b}$ Department of Physics and Astronomy, Shanghai Jiao Tong University, \\ Shanghai 200240, China \\ ${ }^{c}$ Department of Physics, School of Mathematics and Physics, Bohai University, \\ Jinzhou, 121013, China \\ ${ }^{d}$ State Key Laboratory of Theoretical Physics, \\ Institute of Theoretical Physics, Chinese Academy of Sciences, \\ Beijing 100190, China \\ E-mail: kuangxiaomei@sjtu.edu.cn, lpapa@central.ntua.gr, \\ wang_b@sjtu.edu.cn, jianpinwu@gmail.com
}

ABStRaCT: We consider a holographic fermionic system in which the fermions are interacting with a $\mathrm{U}(1)$ gauge field in the presence of a dilaton field in a gravity bulk of a charged black hole with hyperscaling violation. Using both analytical and numerical methods, we investigate the properties of the infrared and ultaviolet Green's functions of the holographic fermionic system. Studying the spectral functions of the system, we find that as the hyperscaling violation exponent is varied, the fermionic system possesses Fermi, non-Fermi, marginal-Fermi and log-oscillating liquid phases. Various liquid phases of the fermionic system with hyperscaling violation are also generated with the variation of the fermionic mass. We also explore the properties of the flat band and the Fermi surface of the non-relativistic fermionic fixed point dual to the hyperscaling violation gravity.

KeYwords: AdS-CFT Correspondence, Holography and condensed matter physics $(\mathrm{AdS} / \mathrm{CMT})$

ARXIV EPRINT: 1409.2945 


\section{Contents}

1 Introduction 1

2 The charged black holes with hyperscaling violation from EinsteinDilaton-Maxwell theory 3

3 The Dirac equation $\quad 7$

$\begin{array}{ll}3.1 \text { The Dirac equation } & 7\end{array}$

3.2 Green's functions 8

4 Low energy behavior and emergent quantum critical behaviour $\quad 8$

4.1 IR Green's function 9

4.2 The analytical expressions of the UV Green's function and the dispersion relation

$\begin{array}{llr}4.3 & \text { Log-periodicity } & 10\end{array}$

5 Properties of the UV Green's function $\quad 11$

5.1 Hyperscaling exponent $\theta$ dependence $\quad 12$

$\begin{array}{lll}5.2 & \text { Mass dependence } & 13\end{array}$

5.2.1 Mass dependence in a gravity bulk with a RN-AdS black hole $\quad 14$

5.2.2 Mass dependence in charged black hole gravity bulk with hyperscaling violation 14

5.3 Failure to generate a dynamical gap in holographic fermionic systems with $\begin{array}{ll}\text { hyperscaling violation } & 15\end{array}$

6 Non-relativistic fermionic fixed point $\quad 16$

$\begin{array}{lll}7 & \text { Conclusions and discussion } & 18\end{array}$

\section{Introduction}

The AdS/CFT conjecture and more generally the gauge/gravity duality offered a new venue of describing many physical systems holographically. The holographic description allows the connection of a $d$-dimensional quantum field theory with its dual gravitational theory that lives in $(d+1)$ dimensions [1-3]. The dual nature of these two theories means that a strong-coupling limit of one of them corresponds to a weak-coupling limit of the other. This is a powerful result since, by employing the gauge/gravity duality, strongly coupled phenomena can be studied using dual gravitational systems in weak coupling.

This holographic description has attracted considerable interest for its potential applications to study strongly coupled systems related to condensed matter (CM) physics. 
One noticeable application of the gauge/gravity application to CM physics is the study of the many-body system at finite charge density. The dual description of such a system is achieved by introducing in the gravity sector, charged fermions probe coupled to the gauge field and exploring the ground state of the holographic system [4-6]. The spectral function of the holographic fermion system was analyzed to study its Fermi surface, low energy excitations and possible types of Fermi and non-Fermi liquids.

In this direction and in an attempt to describe the various phases of a metallic state at low temperatures, a dipole coupling to massless charged fermions was introduced $[7,8]$. Then by studying the modified Dirac equation, it was found that the boundary fermionic propagator produces a spectrum which has vanishing spectral weight at a range of energies around $\omega=0$ without the breaking of any symmetry. Another interesting result was that as the dipole coupling strength was varied, the fluid possessed Fermi, marginal Fermi, non-Fermi liquid phases and an insulating phase which shared similarities with the Mott insulators including the dynamic formation of a gap and spectral weight transfer. These results had stimulated further studies [9-16] on the behaviour of the dipole coupling. A remarkable property was observed in [17] where it was found that there exists a duality between zeroes and poles in holographic systems with massless fermions and a dipole coupling and this property was also verified in [18].

The AdS/CFT correspondence was initially applied to describe holographic fermionic systems in which the space of their gravity sector was described by an AdS geometry. However, in many condensed matter systems, there exist some phase transitions governed by fixed points with Lifshitz dynamical scaling and many non-relativistic fixed points. Therefore, there was a need to formulate the duality principle to describe quantum field theories violating conformal invariance but keeping scale-invariance, having as a gravity dual to a gravity theory with a metric with Lifshitz scaling [19-22]

$$
d s^{2}=-r^{2 z} d t^{2}+\frac{d r^{2}}{r^{2}}+r^{2} d x_{i}^{2}
$$

which is however invariant under the scaling transformation

$$
t \rightarrow \lambda^{z} t, x_{i} \rightarrow \lambda x_{i}, r \rightarrow \lambda^{-1} r
$$

This metric is characterized by a dynamical critical exponent $z \neq 1$, in which with $z=1$ we go back to the AdS metric.

This generalization of the holographic principle from AdS spaces of the gravity sector to Lifshitz spaces had produced interesting results. It was showed that the Lifshitz exponent $z$ in the holographic fermion systems plays an important role in the retarded Green's function $[23,24]$. For a specific value of the critical exponent $z$, the Luttinger's theorem is violated [25] and even a dynamical gap can be generated in the presence of a dipole coupling [26-28].

More recently, a larger class of scaling metrics besides the Lifshitz one was found, by including both Abelian gauge field and a dilaton field in the bulk theory. These metrics with an overall hyperscaling factor which can be considered as an extension of the Lifshitz 
metric have the form [29]

$$
d s^{2}=r^{\frac{-2 \theta}{d}}\left(-r^{2 z} d t^{2}+\frac{d r^{2}}{r^{2}}+r^{2} d x_{i}^{2}\right)
$$

with $z$ and $\theta$ the dynamical critical exponent and the hyperscaling violation exponent respectively [30]. Note that the metric (1.3) transforms as $d s \rightarrow \lambda^{\theta / d} d s$ under the transformation (1.2). Under this scaling the distance is not invariant with a non-zero $\theta$ and according to the AdS/CFT correspondence this indicates a hyperscaling violation in the dual field theory. Thermodynamically in these theories the entropy scales as $T^{(d-\theta) / z}$ while in theories with hyperscaling, the entropy scales as $T^{d / z}$. This implies that the theory with hyperscaling violation brings in an effective dimension $d_{\mathrm{eff}}=d-\theta$. Holographic gravity theories with hyperscaling violation were discussed in [31-33]. It was found in $[34,35]$ that theories with hyperscaling violation in the probe approximation and without the presence of fermions exhibit similarities with the behaviour of a Fermi liquid.

In this work we will consider a holographic fermion system dual to a gravity bulk with hyperscaling violation and with finite charge density. Our aim is to study in details the behaviour of infrared (IR) and ultra violet (UV) Green's functions in an attempt to understand the formation of Fermi surface and the types of the Fermi liquids present in these theories. We will also study the possibility of generating dynamically a gap which will indicate the presence of a Mott insulating phase in theories with hyperscaling violation. Finally, we will explore the spectral function of the non-relativistic fixed point by adding the Lorentz-violating boundary condition into the bulk action.

Holographic study of the fermion system in the gravity dual with hyperscaling violation was discussed in [36]. Their gravitational background was with neutral charge. The fermion system in a charged background was studied in [37]. The UV Green's function in the probe fermions limit was studied numerically and it was found that the increase of the Lifshitz factor $z$ and the hyperscaling factor $\theta$ broadened and smoothed out the sharp peak.

The work is organized as follows. In section 2 we review the charged black hole background with hyperscaling factor and analyze the geometry in the near horizon limit at zero temperature. We set up the formalism describing the equation of motion in the fermionic system in the bulk theory in section 3. In section 4 we analytically investigate the low energy behaviour and emergent quantum critical behavior of the retarded Green function of the dual Fermi operator. In section 5 we numerically study various properties of the UV Green's function. Then we explore the holographic non-relativistic fixed point with Lorentz-violating boundary condition in section 6 . Finally section 7 are our conclusions.

\section{The charged black holes with hyperscaling violation from Einstein- Dilaton-Maxwell theory}

We start with the Einstein-Maxwell-Dilaton action in (3+1)-dimensional spacetime [22]

$$
S_{g}=-\frac{1}{16 \pi G} \int \mathrm{d}^{4} x \sqrt{-g}\left[R-\frac{1}{2}(\partial \phi)^{2}+V(\phi)-\frac{1}{4}\left(e^{\lambda_{1} \phi} F^{\mu \nu} F_{\mu \nu}+e^{\lambda_{2} \phi} \mathcal{F}^{\mu \nu} \mathcal{F}_{\mu \nu}\right)\right] .
$$


The action contains two $\mathrm{U}(1)$ gauge fields coupled to a dilaton field $\phi$. The $\mathrm{U}(1)$ field $A$ with field strength $F_{\mu \nu}$ is required to have a charged black hole solution, while the other gauge field $\mathcal{A}$ with field strength $\mathcal{F}_{\mu \nu}$ and with its coupling to the dilaton field is necessary to generate an anisotropic scaling. We can deduce the equations of motion for all the fields from the above action. The Einstein equation of motion for the metric is

$$
\begin{aligned}
R_{\mu \nu}-\frac{1}{2} R g_{\mu \nu}= & \frac{1}{2} \partial_{\mu} \phi \partial_{\nu} \phi-\frac{V(\phi)}{2} g_{\mu \nu} \\
& +\frac{1}{2}\left[e^{\lambda_{1} \phi}\left(F_{\mu \rho} F_{\nu}^{\rho}-\frac{g_{\mu \nu}}{4} F^{\rho \sigma} F_{\rho \sigma}\right)+e^{\lambda_{2} \phi}\left(\mathcal{F}_{\mu \rho} \mathcal{F}_{\nu}^{\rho}-\frac{g_{\mu \nu}}{4} \mathcal{F}^{\rho \sigma} \mathcal{F}_{\rho \sigma}\right)\right] .
\end{aligned}
$$

The equation of motion for the dilaton field is

$$
\nabla^{2} \phi=-\frac{d V(\phi)}{d \phi}+\frac{1}{4}\left(\lambda_{1} e^{\lambda_{1} \phi} F^{\mu \nu} F_{\mu \nu}+\lambda_{2} e^{\lambda_{2} \phi} \mathcal{F}^{\mu \nu} \mathcal{F}_{\mu \nu}\right)
$$

while the Maxwell equations for the gauge fields read

$$
\begin{aligned}
& \nabla_{\mu}\left(\sqrt{-g} e^{\lambda_{2} \phi} \mathcal{F}^{\mu \nu}\right)=0, \\
& \nabla_{\mu}\left(\sqrt{-g} e^{\lambda_{1} \phi} F^{\mu \nu}\right)=0 .
\end{aligned}
$$

We will introduce a potential of the form

$$
V(\phi)=V_{0} e^{\gamma \phi}
$$

which is very helpful to generate a general Lifshitz solution with hyperscaling violation [37]. Here $\lambda_{1}, \lambda_{2}, \gamma$ and $V_{0}$ are free parameters of the theory to be determined. We consider the following ansatz for the metric

$$
d s_{4}^{2}=r^{-\theta}\left(-r^{2 z} f(r) d t^{2}+\frac{d r^{2}}{r^{2} f(r)}+r^{2}\left(d x^{2}+d y^{2}\right)\right)
$$

Before we proceed with the solution we remark that the two gauge fields appear in the action (2.1) on the same footing. To determine them with their corresponding parameters $\lambda_{i}$ we first decouple the gauge field $F_{\mu \nu}$ which is responsible for the charge of the background black hole and from the field equations (2.2), (2.3) and (2.4) we determine the gauge field $\mathcal{F}_{\mu \nu}$, and then with the use of the Maxwell equation (2.5) we determine the gauge field $F_{\mu \nu}$. Then the solutions are as follows [37]

$$
\begin{aligned}
f & =1-\left(\frac{r_{h}}{r}\right)^{2+z-\theta}+\frac{Q^{2}}{r^{2(z-\theta+1)}}\left[1-\left(\frac{r_{h}}{r}\right)^{\theta-z}\right], \\
\mathcal{F}_{r t} & =\sqrt{2(z-1)(2+z-\theta)} e^{\frac{2-\theta / 2}{\sqrt{2(2-\theta)(z-1-\theta / 2)}} \phi_{0}} r^{1+z-\theta}, \\
F_{r t} & =Q \sqrt{2(2-\theta)(z-\theta)} e^{-\sqrt{\frac{z-1+\theta / 2}{2(2-\theta)}} \phi_{0}} r^{-(z-\theta+1)}, \\
e^{\phi} & =e^{\phi_{0}} r^{\sqrt{2(2-\theta)(z-1-\theta / 2)}} .
\end{aligned}
$$


Here, $r_{h}$ is the radius of horizon satisfying $f\left(r_{h}\right)=0$ and $Q=\frac{1}{16 \pi G} \int e^{\lambda_{1} \phi} F_{r t}$ is the total charge of the black hole. All the parameters in the action depend on the Lifshitz scaling exponent $z$ and hyperscaling violation exponents $\theta$ and they can be written as

$$
\begin{aligned}
\lambda_{1} & =\sqrt{\frac{2(z-1-\theta / 2)}{2-\theta}}, \\
\lambda_{2} & =-\frac{2(2-\theta / 2)}{\sqrt{2(2-\theta)(z-\theta / 2-1)}}, \\
\gamma & =\frac{\theta}{\sqrt{2(2-\theta)(z-1-\theta / 2)}}, \\
V_{0} & =e^{\frac{-\theta \phi_{0}}{\sqrt{2(2-\theta)(z-1-\theta / 2)}}(z-\theta+1)(z-\theta+2) .}
\end{aligned}
$$

Note that we have $z \geq 1$ and $\theta \geq 0$. Especially, the above solution is not valid for $\theta=2$. Also from equations (2.9) and (2.10) we obtain

$$
\begin{aligned}
& \mathcal{A}_{t}=-\mu r_{h}^{2+z-\theta}\left[1-\left(\frac{r}{r_{h}}\right)^{2+z-\theta}\right], \\
& A_{t}=\mu r_{h}^{\theta-z}\left[1-\left(\frac{r_{h}}{r}\right)^{z-\theta}\right],
\end{aligned}
$$

where we have defined

$$
\begin{aligned}
& \mu=\frac{\sqrt{2(z-1)(2+z-\theta)}}{2+z-\theta} e^{\frac{2-\theta / 2}{\sqrt{2(2-\theta)(z-1-\theta / 2)}} \phi_{0}}, \\
& \mu=Q \sqrt{\frac{2(2-\theta)}{z-\theta}} e^{-\sqrt{\frac{z-1+\theta / 2}{2(2-\theta)}} \phi_{0}} .
\end{aligned}
$$

The Hawking temperature of the black hole is

$$
T=\frac{(2+z-\theta) r_{h}^{z}}{4 \pi}\left[1-\frac{(z-\theta) Q^{2}}{2+z-\theta} r_{h}^{2(\theta-z-1)}\right] .
$$

Before proceeding, we would like to remark on the parameters $z$ and $\theta$. First, the background solution given by the equations (2.7)-(2.11) is valid only for $z \geq 1$ and $\theta \geq 0$. The case of $z=1$ and $\theta=0$ corresponds to AdS geometry. Second, the condition $z-\theta \geq 0$ is required to make the chemical potential well-defined in the dual field theory. Third, it is easy to see that $\theta<2$ from equation (2.16). Combining the requirement of the null energy condition $\left(-\frac{\theta}{2}+1\right)\left(-\frac{\theta}{2}+z-1\right) \geq 0$ [37], one can have $\theta \leq 2(z-1)$. Thus, in this charged background, the region of the parameters is

$$
\left\{\begin{array}{l}
0 \leq \theta \leq 2(z-1) \text { for } 1 \leq z<2 \\
0 \leq \theta<2 \text { for } z \geq 2
\end{array}\right.
$$

For convenience, we make the following rescaling

$$
\begin{aligned}
& r \rightarrow r_{h} r, \quad t \rightarrow \frac{t}{r_{h}^{z}}, \quad(x, y) \rightarrow \frac{1}{r_{h}}(x, y), \\
& Q \rightarrow r_{h}^{(z-\theta+1)} Q, \quad A_{t} \rightarrow r_{h} A_{t}, \quad \mathcal{A}_{t} \rightarrow r_{h}^{\theta-z-2} \mathcal{A}_{t} \text {. }
\end{aligned}
$$


With the rescaling above, we can set $r_{h}=1$. In addition, note that $\phi_{0}$ is an integration constant and we will set $\phi_{0}=0$ in the following. With such rescaling, the redshift factor $f(r)$ and the gauge fields $\mathcal{A}_{t}, A_{t}$ can be expressed respectively as,

$$
\begin{aligned}
f & =1-\frac{1+Q^{2}}{r^{z+2-\theta}}+\frac{Q^{2}}{r^{2(z-\theta+1)}}, \\
\mathcal{A}_{t} & =-\mu\left[1-r^{2+z-\theta}\right], \\
A_{t} & =\mu\left[1-\left(\frac{1}{r}\right)^{z-\theta}\right] .
\end{aligned}
$$

and the dimensionless temperature has the form

$$
T=\frac{(2+z-\theta)}{4 \pi}\left[1-\frac{(z-\theta) Q^{2}}{2+z-\theta}\right] .
$$

By setting

$$
Q=\sqrt{\frac{2+z-\theta}{z-\theta}}, \quad \text { i.e., } \quad \mu=\frac{\sqrt{2(2-\theta)(2+z-\theta)}}{z-\theta}
$$

one can obtain the zero-temperature limit, in which the redshift factor $f(r)$ becomes

$$
\left.f(r)\right|_{T=0}=1-\frac{2(z-\theta+1)}{z-\theta} \frac{1}{r^{z-\theta+2}}+\frac{z-\theta+2}{z-\theta} \frac{1}{r^{2(z-\theta+1)}} .
$$

Obviously, in the $r \rightarrow 1$ limit,

$$
\left.f(r)\right|_{T=0, r \rightarrow 1} \simeq(z-\theta+1)(z-\theta+2)(r-1)^{2} \equiv \frac{1}{L_{2}^{2}}(r-1)^{2} .
$$

Therefore, at the zero temperature, we obtain the near horizon geometry $A d S_{2} \times \mathbb{R}^{2}$ with the curvature radius $L_{2} \equiv 1 / \sqrt{(z-\theta+1)(z-\theta+2)}$ of $A d S_{2}$ to depend explicitly on the Lifshitz scaling exponent $z$ and hyperscaling violation exponent $\theta$. So, near the horizon, the metric and the gauge fields are given by

$$
\begin{aligned}
d s^{2} & =\frac{L_{2}^{2}}{\varsigma^{2}}\left(-d \tau^{2}+d \varsigma^{2}\right)+d x^{2}+d y^{2}, \\
\mathcal{A}_{\tau} & =\frac{\notin}{\varsigma}, \quad A_{\tau}=\frac{e}{\varsigma},
\end{aligned}
$$

with $\phi=\mu(2+z-\theta) L_{2}^{2}$ and $e=\mu(z-\theta) L_{2}^{2}$ where we have considered the following scaling limit

$$
r-1=\epsilon \frac{L_{2}^{2}}{\varsigma}, \quad t=\epsilon^{-1} \tau,
$$

with $\epsilon \rightarrow 0, \varsigma$ and $\tau$ to be finite. 


\section{The Dirac equation}

\subsection{The Dirac equation}

To probe the geometry with hyperscaling violation, we consider the following Dirac action including the bulk minimal coupling between the fermion and the gauge field

$$
S_{D}=i \int d^{4} x \sqrt{-g} \bar{\zeta}\left(\Gamma^{a} \mathcal{D}_{a}-m\right) \zeta,
$$

where $\mathcal{D}_{a}=\partial_{a}+\frac{1}{4}\left(\omega_{\mu \nu}\right)_{a} \Gamma^{\mu \nu}-i q A_{a}$. From the above action, we can derive the following Dirac equation in Fourier space

$$
\left(\sqrt{g^{r r}} \Gamma^{r} \partial_{r}-m\right) F-i\left(\omega+q A_{t}\right) \sqrt{g^{t t}} \Gamma^{t} F+i k \sqrt{g^{x x}} \Gamma^{x} F=0 .
$$

In the above equation, we have made a redefinition of $\zeta=\left(-g g^{r r}\right)^{-\frac{1}{4}} \mathcal{F}$ and a Fourier transformation $\mathcal{F}=F e^{-i \omega t+i k_{i} x^{i}}$. In addition, due to the rotational symmetry in $x-y$ plane, we have set $k_{x}=k$ and $k_{y}=0$. Choosing the following gamma matrices

$$
\Gamma^{r}=\left(\begin{array}{cc}
-\sigma^{3} & 0 \\
0 & -\sigma^{3}
\end{array}\right), \Gamma^{t}=\left(\begin{array}{cc}
i \sigma^{1} & 0 \\
0 & i \sigma^{1}
\end{array}\right), \Gamma^{x}=\left(\begin{array}{cc}
-\sigma^{2} & 0 \\
0 & \sigma^{2}
\end{array}\right), \quad \ldots
$$

the Dirac equation becomes

$$
\left[\left(\partial_{r}+m \sqrt{g_{r r}} \sigma^{3}\right)-\sqrt{\frac{g_{r r}}{g_{t t}}}\left(\omega+q A_{t}\right) i \sigma^{2}-(-1)^{I} k \sqrt{\frac{g_{r r}}{g_{x x}}} \sigma^{1}\right] F_{I}=0,
$$

where $I=1,2$. After splitting $F_{I}$ into $F_{I}=\left(\mathcal{A}_{I}, \mathcal{B}_{I}\right)^{T}$, one has

$$
\begin{aligned}
& \left(\partial_{r}+m \sqrt{g_{r r}}\right) \mathcal{A}_{I}-\sqrt{\frac{g_{r r}}{g_{t t}}}\left(\omega+q A_{t}\right) \mathcal{B}_{I}-(-1)^{I} \sqrt{\frac{g_{r r}}{g_{x x}}} k \mathcal{B}_{I}=0, \\
& \left(\partial_{r}-m \sqrt{g_{r r}}\right) \mathcal{B}_{I}+\sqrt{\frac{g_{r r}}{g_{t t}}}\left(\omega+q A_{t}\right) \mathcal{A}_{I}-(-1)^{I} \sqrt{\frac{g_{r r}}{g_{x x}}} k \mathcal{A}_{I}=0 .
\end{aligned}
$$

Defining $\xi_{I} \equiv \frac{\mathcal{A}_{I}}{\mathcal{B}_{I}}$ and $v=\sqrt{\frac{g_{r r}}{g_{t t}}}\left(\omega+q A_{t}\right)$ the above equations can be brought in the form of a flow equation of $\xi_{I}$

$$
\left(\partial_{r}+2 m \sqrt{g_{r r}}\right) \xi_{I}-\left[v+(-1)^{I} k \sqrt{\frac{g_{r r}}{g_{x x}}}\right]-\left[v-(-1)^{I} k \sqrt{\frac{g_{r r}}{g_{x x}}}\right] \xi_{I}^{2}=0 .
$$

For the convenience of numerical calculation later, we can make a transformation $r=1 / u$, so that the flow equation (3.7) can be rewritten as

$$
\left(\sqrt{f} \partial_{u}-2 m u^{\frac{\theta}{2}-1}\right) \xi_{I}+\left[\frac{\tilde{v}}{u}+(-1)^{I} k\right]+\left[\frac{\tilde{v}}{u}-(-1)^{I} k\right] \xi_{I}^{2}=0
$$

where we have redefined $\tilde{v}=\frac{u^{z}}{\sqrt{f}}\left(\omega+q A_{t}\right)$.

Since the IR geometry of the charged geometry with hyperscaling violation is $A d S_{2} \times$ $\mathbb{R}^{2}$, we can easily derive the boundary conditions of $\xi$ at the horizon $r=1$ for $\omega \neq 0[5,6,10]$

$$
\xi_{I}=i
$$

For the case of $\omega=0$, we refer to $[5,6]$. 


\subsection{Green's functions}

In the background with hyperscaling violation in the UV limit, from equation (1.3) we know that $g_{r r}=r^{-\theta-2}, g_{t t}=r^{2 z-\theta}$ and $g_{x x}=g_{y y}=r^{2-\theta}$. Therefore, the Dirac equation (3.4) becomes

$$
\left[\partial_{r}+m r^{-\frac{\theta}{2}-1} \sigma^{3}-r^{-1-z}(\omega+q \mu) i \sigma^{2}-(-1)^{I} k r^{-2} \sigma^{1}\right] F_{I}=0 .
$$

Since we have $\theta<2$ from equation (2.18), in the limit of $r \rightarrow \infty$, equation (3.10) reduces to

$$
\left(\partial_{r}+\frac{m}{r^{\frac{\theta}{2}+1}} \sigma^{3}\right) F_{I} \approx 0
$$

which gives the following solutions

$$
\begin{aligned}
& \mathcal{A}_{I}=a_{I} e^{\frac{2 m}{\theta} r^{-\frac{\theta}{2}}} \simeq a_{I}\left(1+\frac{2 m}{\theta} r^{-\frac{\theta}{2}}+\ldots\right), \\
& \mathcal{B}_{I}=b_{I} e^{-\frac{2 m}{\theta} r^{-\frac{\theta}{2}}} \simeq b_{I}\left(1-\frac{2 m}{\theta} r^{-\frac{\theta}{2}}+\ldots\right) .
\end{aligned}
$$

Thus, at the leading order, $F_{I}$ behaves as

$$
F_{I} \stackrel{r \rightarrow \infty}{\approx} b_{I}\left(\begin{array}{l}
0 \\
1
\end{array}\right)+a_{I}\left(\begin{array}{l}
1 \\
0
\end{array}\right)
$$

which agrees well with the case of zero mass in AdS or Lifshitz-AdS geometry.

In the regime of linear response, the boundary Green's functions can be extracted by $G_{I I}=\frac{a_{I}}{b_{I}}$. At the same time, since

$$
\xi_{I} \equiv \frac{\mathcal{A}_{I}}{\mathcal{B}_{I}}=\frac{a_{I}}{b_{I}}=G_{I},
$$

the boundary retarded Green's functions can be expressed in term of $\xi_{I}$

$$
G(\omega, k)=\left(\begin{array}{cc}
G_{1} & 0 \\
0 & G_{2}
\end{array}\right)=\lim _{r \rightarrow \infty}\left(\begin{array}{cc}
\xi_{1} & 0 \\
0 & \xi_{2}
\end{array}\right) .
$$

Also, from equation (3.8), we can see that the Green function has the following properties

$$
G_{1}(\omega, k ; m)=G_{2}(\omega,-k ; m), \quad G_{I}(\omega, k ;-m)=-\frac{1}{G_{I}(\omega,-k ; m)} .
$$

\section{Low energy behavior and emergent quantum critical behaviour}

As pointed out in section 2, the extremal near horizon geometry of this charged black hole with hyperscaling violation is $A d S_{2} \times \mathbb{R}^{2}$. Thus, we can discuss the retarded Green's function and some related emergent quantum critical behavior by using the matching method [6]. 


\subsection{IR Green's function}

For the near horizon geometry $A d S_{2} \times \mathbb{R}^{2}$, in the limit of $\omega \rightarrow 0$, the Dirac equation is

$$
\varsigma \partial_{\varsigma} F_{I}-\left[m L_{2} \sigma^{3}-(-1)^{I} k L_{2} \sigma^{1}-i \sigma^{2} q e\right] F_{I}=0 .
$$

In the above equation, we have chosen the same Gamma matrices as in equation (3.3) except $\Gamma^{\varsigma}=-\Gamma^{r}$ to reflect the change between the radial coordinate $r$ and the coordinate $\varsigma$. The above equation can be rewritten as

$$
\varsigma \partial_{\varsigma} F_{I}=U F_{I},
$$

where

$$
U=\left(\begin{array}{cc}
m L_{2} & -(-1)^{I} k L_{2}-q e \\
-(-1)^{I} k L_{2}+q e & -m L_{2}
\end{array}\right) .
$$

Therefore, near the $A d S_{2}$ boundary $(\varsigma \rightarrow 0)$, the leading behaviour of $F_{I}$ is

$$
F_{I}=b_{I}^{(0)} v_{-} \varsigma^{-\nu_{I}(k)}+a_{I}^{(0)} v_{+} \varsigma^{\nu_{I}(k)},
$$

where $v_{ \pm}$are real eigenvectors of $U$ and $\pm \nu_{I}(k)$ are eigenvalues in the form

$$
\nu_{I}(k)=\sqrt{\left(m^{2}+k^{2}\right) L_{2}^{2}-q^{2} e^{2}} .
$$

Then the dimension in the IR CFT of the operator $\mathcal{O}_{\vec{k}}$ is given by $\delta_{k}=\frac{1}{2}+\nu_{I}(k)$, which obviously depends on the Lifshitz dynamical critical exponent $z$ and hyperscaling violating exponent $\theta$. We can exactly solve the Dirac equation (4.1) in $A d S_{2}$ and obtain the retarded Green's functions of $\mathcal{O}_{\vec{k}}$ in the dual IR CFT as [6]

$$
\mathcal{G}_{I}(k, \omega)=c(k) e^{-i \pi \nu_{I}(k)} \omega^{2 \nu_{I}(k)} .
$$

\subsection{The analytical expressions of the UV Green's function and the dispersion relation}

In this subsection, we will discuss the case of $\nu_{I}(k)$ being real. The case of the imaginary $\nu_{I}(k)$ will be discussed in the next subsection.

In general, the bulk spacetime can be divided into the inner and outer regions

$$
\begin{aligned}
& \text { Inner : } r-1=\omega \frac{L_{2}^{2}}{\varsigma}, \quad \epsilon<\varsigma<\infty, \\
& \text { Outer : } r-1>\omega \frac{L_{2}^{2}}{\epsilon},
\end{aligned}
$$

where $\omega, \epsilon, \omega \frac{L_{2}^{2}}{\epsilon} \rightarrow 0$, but $\varsigma$ is finite. When $\varsigma \rightarrow 0$ and $\omega / \varsigma \rightarrow 0$, there is a non-zero overlapping region between the inner and outer regions. Thus, within this region, $a_{I}$ and $b_{I}$ in equation (3.14) can be expanded in terms of the power of $\omega$

$$
\begin{aligned}
a_{I} & =\left[a_{I}^{(0)}+\omega a_{I}^{(1)}+\ldots\right]+\left[\tilde{a}_{I}^{(0)}+\omega \tilde{a}_{I}^{(1)}+\ldots\right] \mathcal{G}_{I}(k, \omega), \\
b_{I} & =\left[b_{I}^{(0)}+\omega b_{I}^{(1)}+\ldots\right]+\left[\tilde{b}_{I}^{(0)}+\omega \tilde{b}_{I}^{(1)}+\ldots\right] \mathcal{G}_{I}(k, \omega),
\end{aligned}
$$


so that the UV Green's function can be expressed in terms of the IR Green's function

$$
G_{I}(\omega, k)=K \frac{a_{I}^{(0)}+\omega a_{I}^{(1)}+\mathcal{O}\left(\omega^{2}\right)+\left(\tilde{a}_{I}^{(0)}+\omega \tilde{a}_{I}^{(1)}+\mathcal{O}\left(\omega^{2}\right)\right) \mathcal{G}(k, \omega)}{b_{I}^{(0)}+\omega b_{I}^{(1)}+\mathcal{O}\left(\omega^{2}\right)+\left(\tilde{b}_{I}^{(0)}+\omega \tilde{b}_{I}^{(1)}+\mathcal{O}\left(\omega^{2}\right)\right) \mathcal{G}(k, \omega)},
$$

where $K$ is a constant. Then, we have for $a_{I}^{(0)} \neq 0$

$$
\operatorname{Im} \mathrm{G}_{\mathrm{I}}(\omega, \mathrm{k}) \simeq \mathrm{K} \frac{\mathrm{a}_{\mathrm{I}}^{(0)}}{\mathrm{b}_{\mathrm{I}}^{(0)}}\left(\frac{\tilde{\mathrm{a}}_{\mathrm{I}}^{(0)}}{\mathrm{a}_{\mathrm{I}}^{(0)}}-\frac{\tilde{\mathrm{b}}_{\mathrm{I}}^{(0)}}{\mathrm{b}_{\mathrm{I}}^{(0)}}\right) \mathrm{c}(\mathrm{k}) \omega^{2 \nu_{\mathrm{I}}(\mathrm{k})}
$$

which will bring us the spectral function near small frequency

$$
A(\omega, k)=\operatorname{Im} \operatorname{Tr}[\mathrm{G}] \propto \mathrm{c}(\mathrm{k}) \omega^{2 \nu_{\mathrm{I}}(\mathrm{k})}
$$

with the scaling exponent $2 \nu_{I}(k)$ dependent on the bulk exponent. For $a_{I}^{(0)}=0$, at small $\omega$ and near the Fermi momentum $k_{F}$, to the leading order, one has

$$
G_{I}(\omega, k) \simeq \frac{h_{1}}{\tilde{k}-\frac{\omega}{v_{F}}-h_{2} e^{i \gamma(k)} \omega^{2 \nu_{I}(k)}},
$$

where $v_{F}, h_{1}$ and $h_{2}$ depend on the UV data and are usually determined numerically. From the above equation, it is easy to conclude that the dispersion relation is

$$
\tilde{\omega}(\tilde{k}) \propto \tilde{k}^{\delta}, \quad \text { with } \quad \delta=\left\{\begin{array}{ll}
\frac{1}{2 \nu_{I}\left(k_{F}\right)} & \nu_{I}\left(k_{F}\right)<\frac{1}{2} \\
1 & \nu_{I}\left(k_{F}\right)>\frac{1}{2}
\end{array} .\right.
$$

Usually, the Fermi momentum $k_{F}$ is determined numerically. In the next section, we will study how both Lifshitz exponent $z$ and hyperscaling exponent $\theta$ affect the Fermi surface structure, the dispersion relation and what kind of Fermi liquids they give.

\subsection{Log-periodicity}

Now we move on to study the case of the imaginary $\nu_{I}(k)$, which usually gives a log-periodic oscillatory behaviour of the fermionic systems. When

$$
k^{2}<k_{0}^{2} \equiv \frac{q^{2} e^{2}}{L_{2}^{2}}-m^{2},
$$

$\nu_{I}(k)$ is purely imaginary. Now, to the leading order, the UV Green's function at small $\omega$ becomes

$$
G_{I I}(\omega, k) \simeq \frac{a_{I}^{(0)}+\tilde{a}_{I}^{(0)} c(k) \omega^{-2 i \lambda_{I}(k)}}{b_{I}^{(0)}+\tilde{b}_{I}^{(0)} c(k) \omega^{-2 i \lambda_{I}(k)}},
$$

where we have denoted $\nu_{I}(k)=-i \lambda_{I}(k)$ with

$$
\lambda_{I}(k)=\sqrt{q^{2} e^{2}-\left(m^{2}+k^{2}\right) L_{2}^{2}} .
$$

It is easy to find that the Green's function (4.17) is log-periodic with a period $\tau_{k}=\pi / \lambda_{I}(k)$ for the imaginary $\nu_{I}(k)$. Therefore, we refer to the region (4.16) as the oscillatory region $[6$, 26-28], which depends on the Lifshitz exponent $z$ and hyperscaling exponent $\theta$. Finally, we would like to point out that for spinors, the log-periodic oscillatory behaviour does not mean an instability as it was discussed in [6, 26-28]. 

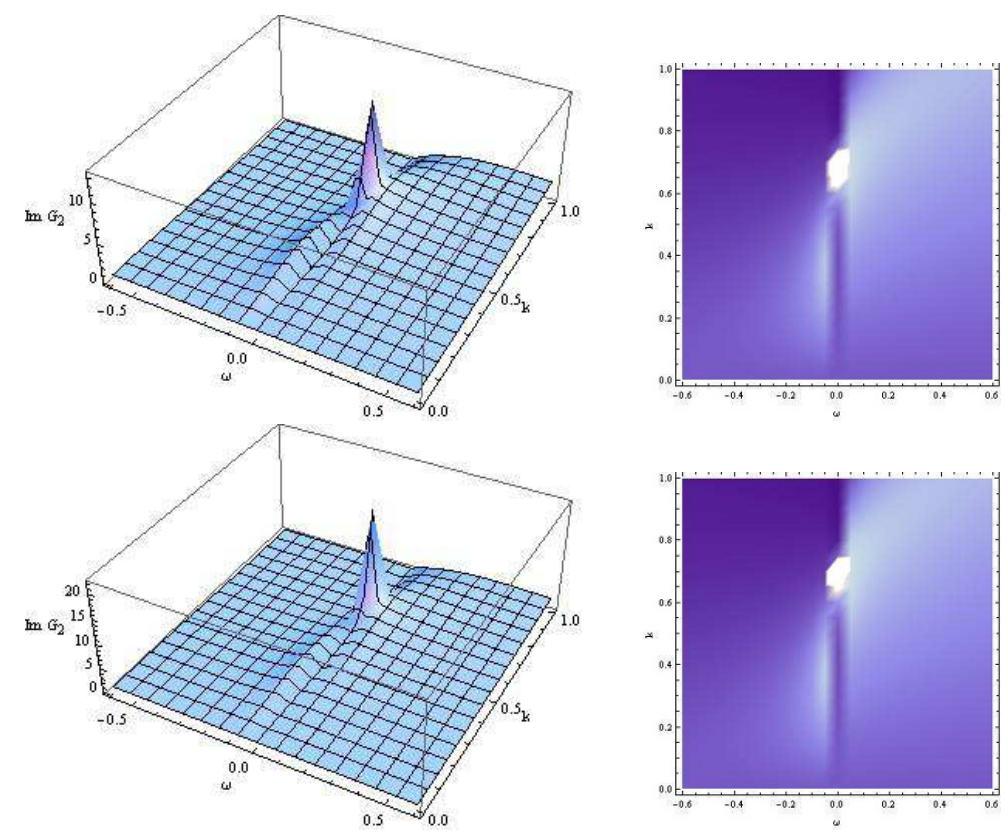

Figure 1. The $3 \mathrm{~d}$ and density plots of $\operatorname{ImG}_{22}(\omega, \mathrm{k})$. The plots above are for $\theta=0$ and the plots below are for $\theta=0.1(q=0.5, m=0$ and $z=1.2)$.

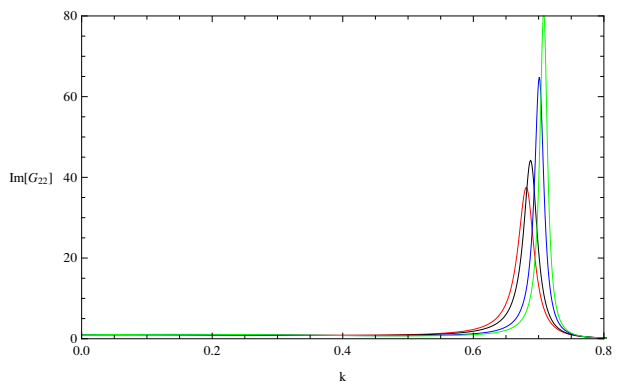

Figure 2. The plot of $\mathrm{ImG}_{22}$ as a function of $k$ for $z=1.2$ and small $\omega$, with different hyperscaling violating exponent $\theta$ (red for $\theta=0$, black for $\theta=0.1$, blue for $\theta=0.3$ and green for $\theta=0.4$ ). Here $q=0.5$ and $m=0$.

\section{Properties of the UV Green's function}

Now, we turn to study the properties of the UV Green's function by using numerical methods. Previous studies on the effects of the Lifshitz exponent $z$ to the holographic fermionic systems showed that the Lifshitz scaling exponent $z$ gives a clear peak in the retarded Green's function in defining a Fermi surface and revealing its quasi-particle behaviour. Here, we will mainly focus on the effects of hyperscaling exponent $\theta$. In figure 1 , one can notice that in the hyperscaling violation gravity, the quasi-particle-peak becomes wider than that in RN-AdS background as pointed out in [37]. 

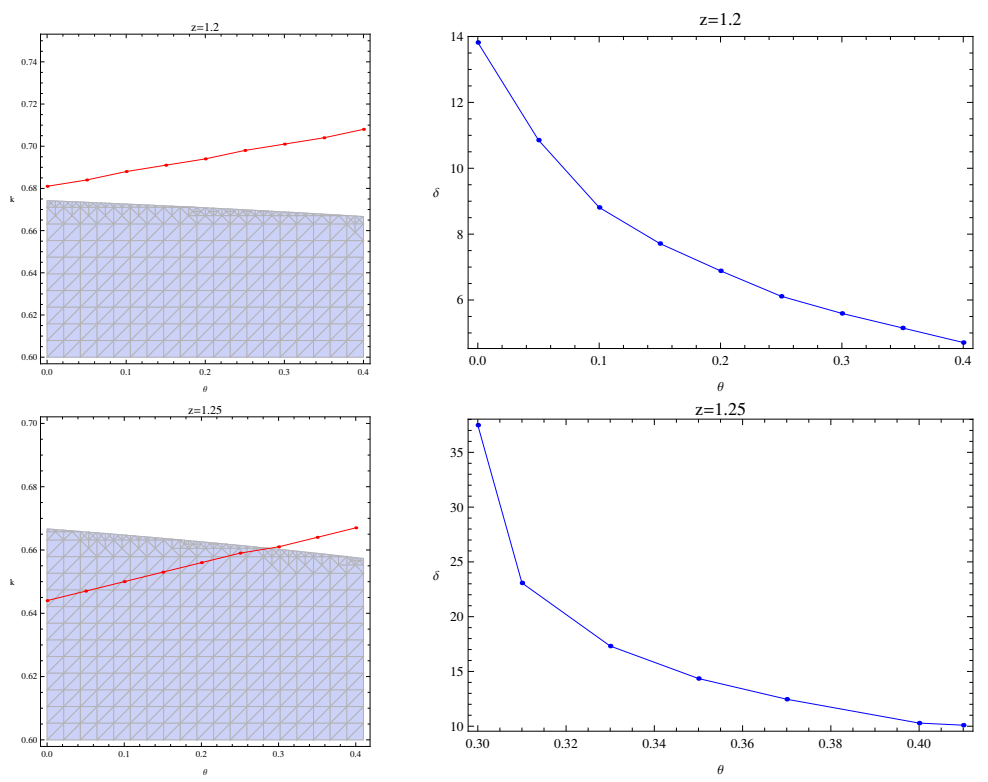

Figure 3. Left plots: the red line is the relation between the hyperscaling exponent $\theta$ and the location of the peak for $\omega \rightarrow 0$ for fixed Lifshitz exponent $z$. The blue shade in the $k-\theta$ space indicates the unstable oscillatory region and above it is the stable region. Right plots: the relation between the hyperscaling exponent $\theta$ and the scaling exponent $\delta$ of dispersion relation for fixed Lifshtiz exponent $z$. Here, $q=0.5, m=0$. In addition, $z=1.2$ in the plot above and $z=1.25$ in the plot below.

\subsection{Hyperscaling exponent $\theta$ dependence}

Now, we will study how the Fermi momentum $k_{F}$ and the scaling exponent $\delta$ of the dispersion relation depend on the hyperscaling exponent $\theta$ for fixed $q, z$ and zero mass of the fermions and what types of Fermi liquids we get depending on the value of $\theta$.

We show the Green's function with the momentum near zero frequency in hyperscaling violation gravity in figure 2 . We find that for fixed $z=1.2$, larger $\theta$ corresponds to higher Fermi momentum, indicating that the hyperscaling exponent $\theta$ has different effects compared to the Lifshitz scaling exponent $z$. This can be explained by the fact that the non-zero hyperscaling exponent introduces an effective dimension of the theory $d_{\mathrm{eff}}=d-\theta$. So larger $\theta$ means lower effective dimension which calls for higher Fermi momentum. This dependence of Fermi momentum on the dimension with minimal coupling was first disclosed and understood in [11].

In the left plot in figure 3 , the relation between the hyperscaling exponent $\theta$ and the location of the peak for $\omega \rightarrow 0$ is presented. In the $k-\theta$ space the region in the blue shade is the log-periodic oscillatory region $[5,6]$. When the location of the peak falls in the region above, it indicates a Fermi surface. But when the location is in the oscillatory region, the peak loses the meaning of Fermi surfaces. From the left plot in figure 3, we find that in the range of allowed $\theta$, all the peaks are located in the region above for $z=1.2$, but for $z=1.25$, when $\theta \leq \theta_{c} \simeq 0.3$, the peak begins to enter the oscillatory regime and then it loses its meaning as Fermi surface. Furthermore, one can find that in the range of allowed $\theta$, when $z \leq 1.21$, all the peaks lie outside the oscillatory region corresponding to a Fermi 


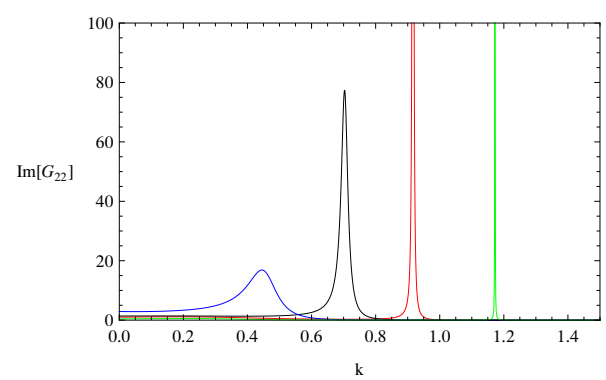

Figure 4. The plot of $\mathrm{ImG}_{22}$ as a function of $k$ in the RN-AdS black hole for tiny $\omega$, with different $m$ (blue for $m=0.4$, black for $m=0.1$, red for $m=0$ and green for $m=-0.2$ ). Here $q=0.5$.
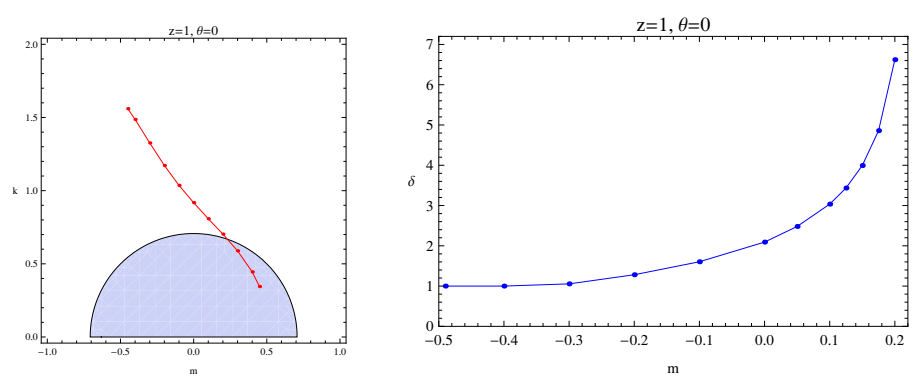

Figure 5. Left plots: the relation between the mass $m$ and the location of the peak for $\omega \rightarrow 0$ in the RN-AdS black hole. The shade region is the oscillatory region. Right plots: the scaling exponent $\delta$ of dispersion relation as a function of $m$ in the RN-AdS black hole. Here, $q=0.5$.

liquid. But for $z \geq 1.22$, the peaks begin to enter the oscillatory region when $\theta$ is smaller than some critical value $\theta_{c}$. This indicates the existence of a marginal Fermi liquid.

Once the Fermi momentum $k_{F}$ is worked out numerically, the dispersion relation can be determined by equation (4.15). Obviously, from the right plots in figure 3 , the exponent $\delta$ of the dispersion relation decreases rapidly as the hyperscaling exponent $\theta$ becomes larger due to the decrease the effective dimension of the dual theory, which is consistent with the dependence of dispersion relation on the dimension discussed in [11]. This indicates that with the increase of $\theta$, it shows smaller degree of deviating from the Landau Fermi liquid phase to a non-Fermi liquid phase.

\subsection{Mass dependence}

It is well known that the types of fermion liquids and possible transition from Fermi to non-fermi liquids depend also on the fermion mass in the holographic fermionic systems [6, 38, 39]. For comparison, we first present the results of the mass dependence in a fermionic system in a Reissner-Nordström-AdS (RN-AdS) black hole background and then we will discuss the mass dependence in the charged black hole with hyperscaling violation. 


\subsubsection{Mass dependence in a gravity bulk with a RN-AdS black hole}

The UV Green's function in the gravity bulk with a RN-AdS black hole is [5, 6]

$$
G(\omega, k)=\lim _{u \rightarrow 0} u^{-2 m}\left(\begin{array}{cc}
\xi_{1} & 0 \\
0 & \xi_{2}
\end{array}\right) .
$$

For the purpose of numerical calculation, we make a transformation $G_{I I}=u^{-2 m} \xi_{I}$, so that one has

$$
\left(\sqrt{f} \partial_{u}+2 m u^{-1} \sqrt{f}-2 m u^{-1}\right) G_{I I}+\left[\frac{\tilde{v}}{u}+(-1)^{I} k\right] u^{-2 m}+\left[\frac{\tilde{v}}{u}-(-1)^{I} k\right] u^{2 m} G_{I I}^{2}=0 .
$$

Solving the above equation with the boundary condition (3.9), one can directly read off the UV boundary Green's function $G_{I I}$.

In figure 4 , we plot the Green's function $\mathrm{ImG}_{22}$ vs. $k$ for tiny $\omega$ with different $m$. One can observe that with the decrease of $m$, the quasi-particle-peak becomes sharper and the Fermi momentum $k_{F}$ is larger. Furthermore, we present the relation between $m$ and the location of the peak for $\omega \rightarrow 0$ in the left plot in figure 5. One finds that the Fermi momentum $k_{F}$ almost linearly decreases with the increase of $m$. When $m>0.2$, the quasi-particle-peak begins to enter into the oscillatory region and loses its meaning of Fermi surface.

After Fermi momentum $k_{F}$ has been worked out numerically, we can use equation (4.15) to calculate the scaling exponent $\delta$ of the dispersion relation. The result is presented in the right plot in figure 5 where one observes that with the decrease of $m$, the scaling exponent $\delta$ decreases. When $m \leq-0.4, \delta=1$, which is a linear dispersion relation. It indicates that there is a transition from non-Fermi liquid to Fermi liquid as the $m$ decreases in holographic fermionic system with a RN-AdS black hole in its dual gravity bulk.

\subsubsection{Mass dependence in charged black hole gravity bulk with hyperscaling violation}

Now, we turn to study the mass dependence in charged black hole gravity bulk with hyperscaling violation. The relations between $m$ and the location of the peak for $\omega \rightarrow 0$ as well as the scaling exponent $\delta$ as a function of $m$ with different $z, \theta$ are presented in figure 6 . The characteristics of the mass dependence in charged black hole gravity bulk with hyperscaling violation are summarized as follows

- For small Lifshitz exponent $z$ and hyperscaling exponent $\theta$, the Fermi momentum $k_{F}$ almost linearly decreases with the increase of $m$ as that in RN-AdS black hole bulk (the above two plots in the left plots in figure 6). In addition, with the decrease of $m$, the scaling exponent $\delta$ decreases and there is a transition from non-Fermi liquid to Fermi liquid as the $m$ decreases (the above two plots in the right of figure 6).

- From the bottom plot in the left of figure 6, we can see that for large Lifshitz exponent $z$ and hyperscaling exponent $\theta$, the Fermi momentum $k_{F}$ still linearly decreases with the increase of $m$ in the region of large $m$. However it does not persist when $m$ 

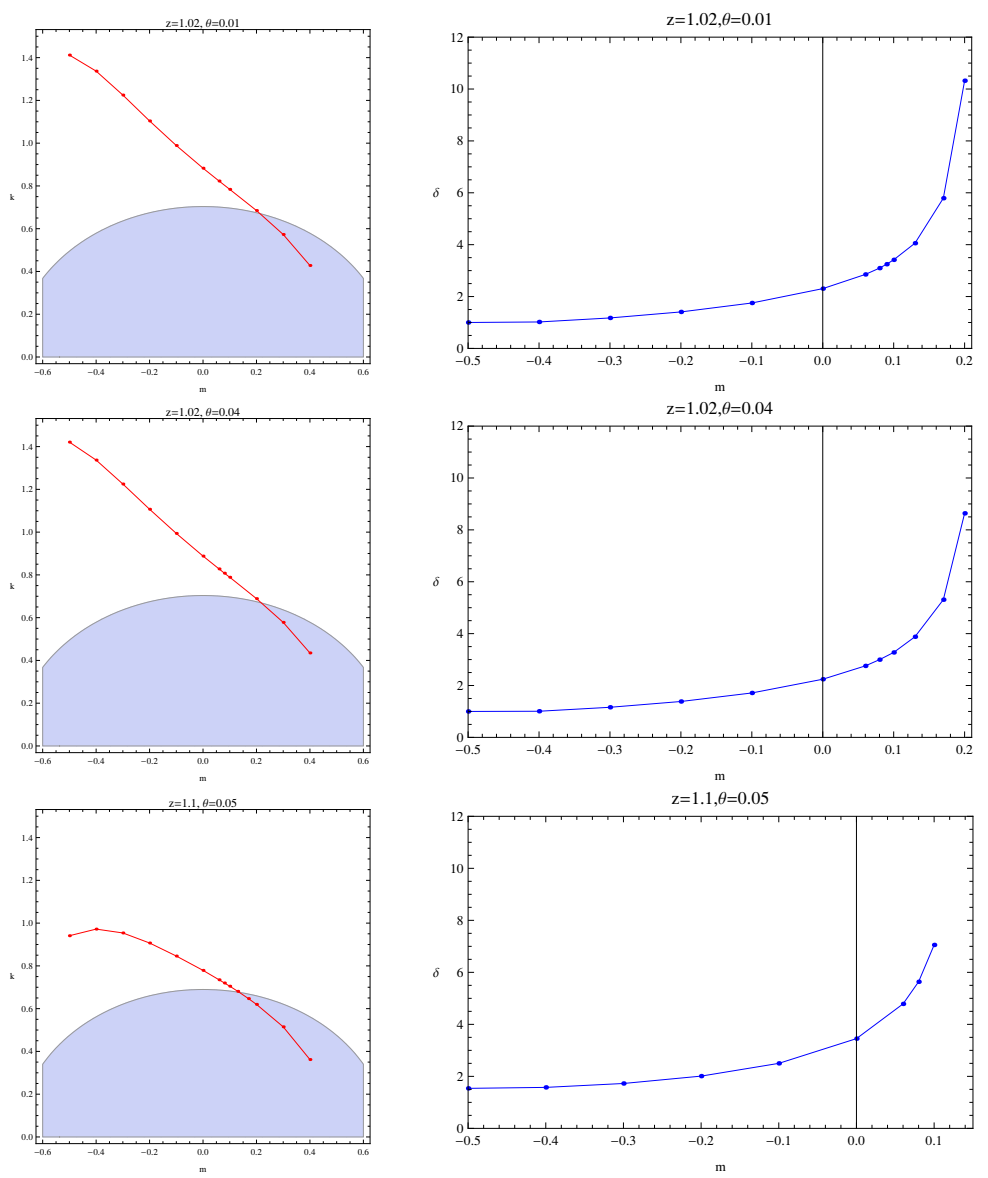

Figure 6. Left plots: the relation between the mass $m$ and the location of the peak for $\omega \rightarrow 0$ with different $z$ and $\theta$. The shade region is the oscillatory region. Right plots: the scaling exponent $\delta$ of dispersion relation as a function of $m$ with different $z$ and $\theta$. Here, $q=0.5$.

approaches the low bound $(m=-0.5)$. Also, in the region of $m \in(-0.5,0.5)$, the scaling exponent $\delta$ is always larger than 1 , which indicates that it is a non-Fermi liquid (the bottom plot in the right of figure 6).

\subsection{Failure to generate a dynamical gap in holographic fermionic systems with hyperscaling violation}

We showed that the variation of the hyperscaling factor $\theta$ can generate various liquid phases like Fermi liquids, non-Fermi liquids, marginal-Fermi liquids and log-oscillatory. The question is if in a holographic fermionic system with hyperscaling violation a gap can be dynamically generated indicating the presence of a Mott insulating phase. In the allowed region (2.18) of $z$ and $\theta$, we numerically obtained the density plot of the Green function $G_{22}$, but we can not see the generated gap near the zero frequency. Two samples of our density plots are showed in figure 7 where we have set $q=0.5$ and $m=0$. We have checked also that other choices of $q$ and $m$ can not generate the Mott gap. It has been firstly proposed in $[7,8]$ that a dipole coupling between the fermions and gauge field mimics 

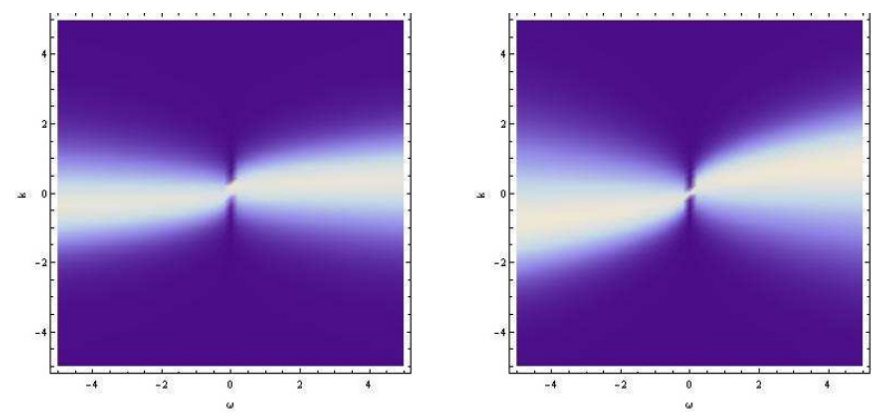

Figure 7. Samples of density plots of the Green function $G_{22}$. Here we set $q=0.5$ and $m=0$. The left plot is for $z=3$ and $\theta=1.5$ while the right one is for $z=2$ and $\theta=1.99$.

doping in the Hubbard model and large enough dipole coupling strength could introduce a Mott gap phase in AdS black hole from holography. Then, it has been addressed in [17] that the pseudo-gap phase can be observed by studying the poles and zeros duality through the $\operatorname{det} G_{R}$. In order to have a complete study of the liquid phases in holographic Fermi systems beyond the dual AdS geometry, it is natural to introduce the dipole coupling in the gravity background with the hyperscaling violation. ${ }^{1}$ The complete study of the effect of the hyperscaling violation on the liquid phase transition between Fermi Liquid, non-Fermi liquids, pseudo-gap and Mott gap is under preparation.

\section{Non-relativistic fermionic fixed point}

Our studies above were focused on the dual relativistic field theory which corresponds to considering the bulk action with the Lorentz covariance boundary term as

$$
S_{\mathrm{bdy}}=\frac{i}{2} \int_{\partial \mathcal{M}} d^{3} x \sqrt{-g g^{r r}} \bar{\zeta} \zeta
$$

In this section, taking into account that our bulk gravity is not boost invariant, we intend to explore some properties of the non-relativistic fixed point by adding a Lorentz violating boundary term into bulk action (3.1)

$$
S_{\mathrm{bdy}}=\frac{1}{2} \int_{\partial \mathcal{M}} d^{3} x \sqrt{-g g^{r r}} \bar{\zeta} \Gamma^{1} \Gamma^{2} \zeta
$$

This boundary term was first proposed in [40], where the authors observed that the spectral function of the dual holographic non-relativistic system showed a flat band of gapless excitation. According to the analysis in $[40,41]$, the retarded Green function of nonrelativistic fixed point can be related to Green functions of the relativistic fixed point as

$$
G_{R}=\left(\begin{array}{ll}
\frac{2 G_{1} G_{2}}{G_{1}+G_{2}} & \frac{G_{1}-G_{2}}{G_{1}+G_{2}} \\
\frac{G_{1}-G_{2}}{G_{1}+G_{2}} & \frac{-2}{G_{1}+G_{2}}
\end{array}\right)
$$

\footnotetext{
${ }^{1}$ We have benefited from discussions we had with Philip Phillips on this point.
} 

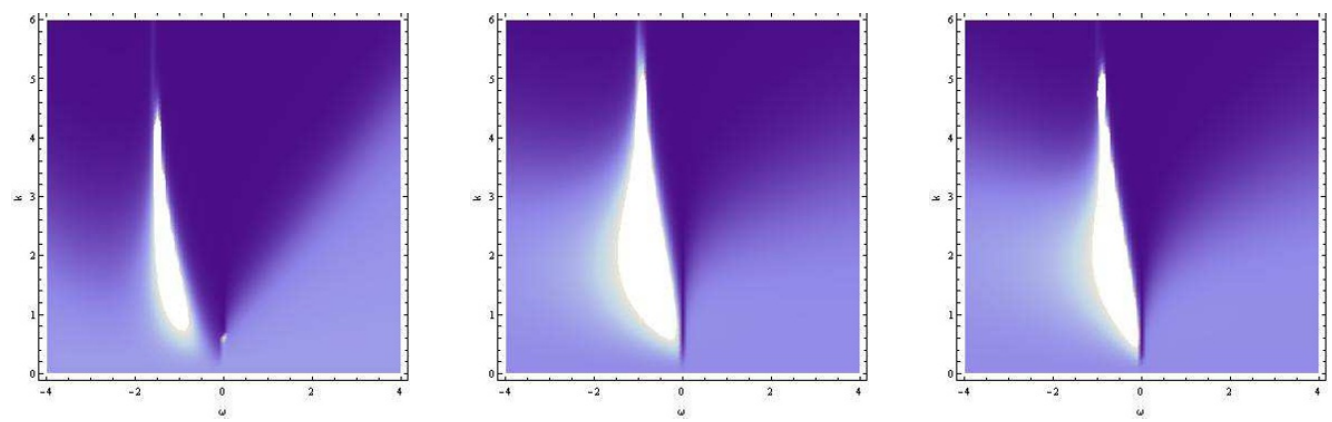

Figure 8. Density plots of the spectral function of non-relativistic fixed point. Here we set $q=0.5$ and $m=0$. The parameters of the plots from left to right are: $z=1 ; \theta=0, z=2 ; \theta=0$ and $z=2 ; \theta=1.5$.

which is off-diagonal with $\operatorname{det} G_{R}=-1$ and its eigenvalue $\lambda_{ \pm}$can be expressed in terms of $G_{I}$ as

$$
\lambda_{ \pm}=\frac{G_{1} G_{2}-1 \pm \sqrt{1+G_{1}^{2}+G_{2}^{2}+G_{1}^{2} G_{2}^{2}}}{G_{1}+G_{2}} .
$$

For simplicity, we will focus on $m=0$ in this section. Then we have $G_{1}=-1 / G_{2}$ from the symmetry (3.17). Thus, the spectral function has the form

$$
A_{N R}(\omega, k)=\operatorname{Im} \operatorname{Tr}\left[\mathrm{G}_{\mathrm{R}}\right]=\operatorname{Im}\left[\frac{4 \mathrm{G}_{\mathrm{I}}}{1-\mathrm{G}_{\mathrm{I}}^{2}}\right] .
$$

Thus we can obtain the spectral function of the non-relativistic fermionic fixed point through extracting the Green function $G_{I}$ by solving the flow equation (3.8).

In figure 8, we show the results of the spectral function $A_{N R}(\omega, k)$ with samples of exponents. For comparison, in the left plot, we reproduced the spectral function for RN-AdS gravity. Then we turned on the dynamical exponent and hyperscaling violation exponent. We found in hyperscaling violation gravity, that the non-relativistic fixed point also presented a flat band with the the similar property of poles distributing continuously at a finite interval of momenta as that in RN-AdS gravity. Namely, the finite band is mildly dispersed at low momentum, but it shows strong peak at high momentum due to the fact that the high momentum modes sit outside the lightcone and can't decay [40].

Furthermore we observed that at large enough momentum the flat band shifts to the frequency $\omega$ which depends on $z$ and $\theta$. This is because the frequency $\omega$ is measured relative to the chemical potential. The flat band corresponds to some zero modes in the Minkowski vaccum, with the vanishing absolute energy of Fermion characterized by $\omega_{\text {eff }}=\omega+q A_{t}$ in the Dirac equation on the boundary. From equation (2.22), it is clear that $A_{t}=\mu$ at the boundary, so that the frequency $\omega$ is related to the chemical potential with $\mu=\frac{\sqrt{2(2-\theta)(2+z-\theta)}}{z-\theta}$ in equation (2.24), which is determined by both the dynamical exponent and hyperscaling violation exponent.

We move on to explore the Fermi momentum and the effect of flat band in the nonrelativistic fixed point. Before processing, it is necessary to point out some features of 


\begin{tabular}{|c|c|c|c|c|c|}
\hline$\theta$ & 0 & 0.1 & 0.2 & 0.3 & 0.4 \\
\hline$k_{F}(R)$ & 0.681 & 0.688 & 0.694 & 0.701 & 0.708 \\
\hline$k_{F}(N R)$ & 0.44 & 0.456 & 0.47 & 0.485 & 0.498 \\
\hline
\end{tabular}

Table 1. The Fermi momentum changes with the hyperscaling exponent with fixed dynamical exponent $z=1.2$ for relativistic and non-relativistic fixed point.

the spectral function (6.5) with massless Dirac field. It was found in [42] that in RN-AdS background the spectral function of the non-relativistic fermionic fixed point has the same scaling behavior at low frequency and dispersion relation expression near the Fermi surface as the relativistic case. Then following $[6,42]$, we can easily obtain that in the hyperscaling violation gravity, the scaling of $A_{N R}(\omega, k)$ near small $\omega$ is the same as the relativistic $A(\omega, k)$ in equation (4.13) and the dispersion relation of $A_{N R}(\omega, k)$ coincides with equation (4.15). Thus, we can numerically determine the Fermi momentum and employ eq. (4.15) to get the dispersion relation of the non-relativistic fixed point.

We give the values of the Fermi momentum with $m=0$ and $q=0.5$ in table 1 . To compare, we set the same exponents for the non-relativistic and relativistic cases and we have chosen $z$ and $\theta$ as that in figure 2. From the table, we see that similar to the relativistic case, the Fermi momentum for non-relativistic case also increases as the hyperscaling violation exponent. However, with any $z$ and $\theta$, the Fermi momentum for non-relativistic case is lower than that in the relativistic case, meaning that the Fermi surface is suppressed by the flat band. This phenomenon was also observed in RN-AdS gravity [41] and charged dilaton gravity [12]. It would be interesting to consider the case with massive fermions.

\section{Conclusions and discussion}

We have studied the features of the fermionic response in a holographic system with a charged black hole with hyperscaling violation in the bulk. Since the near horizon geometry is $A d S_{2}$, we followed the matching method of [6] to obtain the analytical expressions of the UV Green's function and the dispersion relation, which have a similar form as that in RN-AdS black hole [6] and charged Lifshitz background [26-28]. However, since both the Fermi momentum $k_{F}$ and the dimension $\delta_{k}$ in the IR CFT depends on the Lifshitz dynamical critical exponent and hyperscaling violating exponent, the dispersion relation also varies with the two exponents.

We numerically determined the Fermi momentum $k_{F}$ to obtain a relation between the scaling exponent $\delta$ of the dispersion relation and the hyperscaling violating exponent $\theta$. We found that for the case of fixed $q, z$ and zero mass of the fermions, the exponent $\delta$ decreases rapidly as the hyperscaling exponent becomes larger. This indicates that with the increase of $\theta$, the degree of deviation from the Landau Fermi liquid becomes smaller.

Since the boundary Green's function depends on the mass of the fermions, we studied the mass dependence. We found that for small Lifshitz exponent $z$ and hyperscaling exponent $\theta$, with the increase of $m$, the Fermi momentum $k_{F}$ almost linearly decreases as that in RN-AdS black hole and the scaling exponent $\delta$ decreases. We found that there is a 
transition from non-Fermi liquid to Fermi liquid as the $m$ decreases. For large $z$ and $\theta$, the Fermi momentum $k_{F}$ still linearly decreases with the increase of $m$ in the region of large $m$, but this does not hold when $m$ approaches the low bound $(m=-0.5)$. Also, in the region of $m \in(-0.5,0.5)$, the scaling exponent $\delta$ is always larger than 1 , which indicates that it is always in a non-Fermi liquid phase.

We looked for the possibility to generate dynamically a gap indicating the presence of a Mott insulating phase. In the allowed region of $z$ and $\theta$ values we numerically obtained the density plot of the Green function $G_{22}$, but we failed to observe a generation of a gap near the zero frequency. We have also checked that other choices of values of $q$ and $m$ could not generate the Mott gap. This indicates that in order to generate a Mott gap we need to introduce another scale in the fermionic system, such as a dipole moment.

Finally, we added a Lorentz-violating boundary term into the bulk action and investigated the holographic non-relativistic fixed point dual to the hyperscaling violation gravity bulk. Similar to the case in RN-AdS black hole [40], we also observed a flat band of gapless excitation which suppressed the Fermi momentum. Furthermore, here the spectral function at high momentum shifted to frequency dependent on the dynamical exponent and hyperscaling violation exponent, because in the flat band state, the frequency is measured relative to the chemical potential determined by the two exponents in the form of equation (2.24).

It would be interesting to extend this study into a system with a dipole coupling between the gauge field and the fermions and see how the hyperscaling violation imprints on the generation of an insulating phase both in the relativistic and non-relativistic fixed points. Another interesting direction would be to study the case of non-zero temperature. In this way we can study how the phenomena and features disclosed due to the presence of hyperscaling violation would complement the behaviour of quantum liquids and their realization in condensed matter physics.

\section{Acknowledgments}

We thank Philip Phillips for valuable discussions. X. M. K. and E. P. are supported by ARISTEIA II action of the operational programme education and long life learning which is co-funded by the European Union (European Social Fund) and National Resources. B. W. and J. P. W. are supported by the Natural Science Foundation of China. J. P. Wu is also supported by Program for Liaoning Excellent Talents in University (No. LJQ2014123).

Open Access. This article is distributed under the terms of the Creative Commons Attribution License (CC-BY 4.0), which permits any use, distribution and reproduction in any medium, provided the original author(s) and source are credited.

\section{References}

[1] J.M. Maldacena, The large- $N$ limit of superconformal field theories and supergravity, Int. J. Theor. Phys. 38 (1999) 1113 [hep-th/9711200] [INSPIRE].

[2] S.S. Gubser, I.R. Klebanov and A.M. Polyakov, A semiclassical limit of the gauge/string correspondence, Nucl. Phys. B 636 (2002) 99 [hep-th/0204051] [INSPIRE]. 
[3] E. Witten, Anti-de Sitter space and holography, Adv. Theor. Math. Phys. 2 (1998) 253 [hep-th/9802150] [INSPIRE].

[4] S.-S. Lee, A non-Fermi liquid from a charged black hole: a critical Fermi ball, Phys. Rev. D 79 (2009) 086006 [arXiv:0809.3402] [InSPIRE].

[5] H. Liu, J. McGreevy and D. Vegh, Non-Fermi liquids from holography, Phys. Rev. D 83 (2011) 065029 [arXiv: 0903.2477] [inSPIRE].

[6] T. Faulkner, H. Liu, J. McGreevy and D. Vegh, Emergent quantum criticality, Fermi surfaces and $A d S_{2}$, Phys. Rev. D 83 (2011) 125002 [arXiv:0907.2694] [INSPIRE].

[7] M. Edalati, R.G. Leigh and P.W. Phillips, Dynamically generated Mott gap from holography, Phys. Rev. Lett. 106 (2011) 091602 [arXiv: 1010.3238] [INSPIRE].

[8] M. Edalati, R.G. Leigh, K.W. Lo and P.W. Phillips, Dynamical gap and cuprate-like physics from holography, Phys. Rev. D 83 (2011) 046012 [arXiv: 1012.3751] [INSPIRE].

[9] D. Guarrera and J. McGreevy, Holographic Fermi surfaces and bulk dipole couplings, arXiv: 1102.3908 [INSPIRE].

[10] J.P. Wu, Holographic fermions in charged Gauss-Bonnet black hole, JHEP 07 (2011) 106 [arXiv: 1103.3982] [INSPIRE].

[11] X.-M. Kuang, B. Wang and J.-P. Wu, Dipole coupling effect of holographic fermion in the background of charged Gauss-Bonnet AdS black hole, JHEP 07 (2012) 125 [arXiv: 1205.6674] [INSPIRE].

[12] X.-M. Kuang, B. Wang and J.-P. Wu, Dynamical gap from holography in the charged dilaton black hole, Class. Quant. Grav. 30 (2013) 145011 [arXiv:1210.5735] [INSPIRE].

[13] L.Q. Fang, X.-H. Ge and X.-M. Kuang, Holographic fermions with running chemical potential and dipole coupling, Nucl. Phys. B 877 (2013) 807 [arXiv:1304.7431] [INSPIRE].

[14] J.-P. Wu, Some properties of the holographic fermions in an extremal charged dilatonic black hole, Phys. Rev. D 84 (2011) 064008 [arXiv:1108.6134] [inSPIRE].

[15] W.-J. Li and J.-P. Wu, Holographic fermions in charged dilaton black branes, Nucl. Phys. B 867 (2013) 810 [arXiv:1203.0674] [INSPIRE].

[16] W.-J. Li, R. Meyer and H.-B. Zhang, Holographic non-relativistic fermionic fixed point by the charged dilatonic black hole, JHEP 01 (2012) 153 [arXiv:1111.3783] [INSPIRE].

[17] J. Alsup, E. Papantonopoulos, G. Siopsis and K. Yeter, Duality between zeroes and poles in holographic systems with massless fermions and a dipole coupling, arXiv:1404.4010 [INSPIRE].

[18] G. Vanacore and P.W. Phillips, Minding the gap in holographic models of interacting fermions, arXiv:1405.1041 [INSPIRE].

[19] S. Kachru, X. Liu and M. Mulligan, Gravity duals of Lifshitz-like fixed points, Phys. Rev. D 78 (2008) 106005 [arXiv:0808.1725] [INSPIRE].

[20] M. Taylor, Non-relativistic holography, arXiv:0812.0530 [INSPIRE].

[21] D.-W. Pang, A note on black holes in asymptotically Lifshitz spacetime, Commun. Theor. Phys. 62 (2014) 265 [arXiv: 0905.2678] [inSPIRE].

[22] J. Tarrio and S. Vandoren, Black holes and black branes in Lifshitz spacetimes, JHEP 09 (2011) 017 [arXiv:1105.6335] [INSPIRE]. 
[23] U. Gürsoy, E. Plauschinn, H. Stoof and S. Vandoren, Holography and ARPES sum-rules, JHEP 05 (2012) 018 [arXiv:1112.5074] [INSPIRE].

[24] M. Alishahiha, M.R. Mohammadi Mozaffar and A. Mollabashi, Fermions on Lifshitz background, Phys. Rev. D 86 (2012) 026002 [arXiv:1201.1764] [INSPIRE].

[25] L.Q. Fang, X.-H. Ge and X.-M. Kuang, Holographic fermions in charged Lifshitz theory, Phys. Rev. D 86 (2012) 105037 [arXiv:1201.3832] [InSPIRE].

[26] J.-P. Wu, Holographic fermions on a charged Lifshitz background from Einstein-dilaton-Maxwell model, JHEP 03 (2013) 083 [INSPIRE].

[27] J.-P. Wu, Emergence of gap from holographic fermions on charged Lifshitz background, JHEP 04 (2013) 073 [INSPIRE].

[28] J.-P. Wu, The charged Lifshitz black brane geometry and the bulk dipole coupling, Phys. Lett. B 728 (2014) 450 [inSPIRE].

[29] C. Charmousis, B. Gouteraux, B.S. Kim, E. Kiritsis and R. Meyer, Effective holographic theories for low-temperature condensed matter systems, JHEP 11 (2010) 151 [arXiv: 1005.4690] [INSPIRE].

[30] D.S. Fisher, Scaling and critical slowing down in random-field Ising systems, Phys. Rev. Lett. 56 (1986) 416 [INSPIRE].

[31] X. Dong, S. Harrison, S. Kachru, G. Torroba and H. Wang, Aspects of holography for theories with hyperscaling violation, JHEP 06 (2012) 041 [arXiv: 1201.1905] [INSPIRE].

[32] B.S. Kim, Hyperscaling violation: a unified frame for effective holographic theories, JHEP 11 (2012) 061 [arXiv: 1210.0540] [INSPIRE].

[33] M. Alishahiha, A.F. Astaneh and M.R.M. Mozaffar, Thermalization in backgrounds with hyperscaling violating factor, Phys. Rev. D 90 (2014) 046004 [arXiv: 1401.2807] [INSPIRE].

[34] M. Edalati, J.F. Pedraza and W. Tangarife Garcia, Quantum fluctuations in holographic theories with hyperscaling violation, Phys. Rev. D 87 (2013) 046001 [arXiv:1210.6993] [INSPIRE].

[35] M. Edalati and J.F. Pedraza, Aspects of current correlators in holographic theories with hyperscaling violation, Phys. Rev. D 88 (2013) 086004 [arXiv: 1307.0808] [INSPIRE].

[36] Z. Fan, Holographic fermions in asymptotically scaling geometries with hyperscaling violation, Phys. Rev. D 88 (2013) 026018 [arXiv: 1303.6053] [INSPIRE].

[37] M. Alishahiha, E. O Colgain and H. Yavartanoo, Charged black branes with hyperscaling violating factor, JHEP 11 (2012) 137 [arXiv:1209.3946] [INSPIRE].

[38] M. Cubrovic, J. Zaanen and K. Schalm, String theory, quantum phase transitions and the emergent Fermi-liquid, Science 325 (2009) 439 [arXiv:0904.1993] [INSPIRE].

[39] N. Iqbal and H. Liu, Real-time response in AdS/CFT with application to spinors, Fortsch. Phys. 57 (2009) 367 [arXiv: 0903. 2596] [INSPIRE].

[40] J.N. Laia and D. Tong, A holographic flat band, JHEP 11 (2011) 125 [arXiv:1108.1381] [INSPIRE].

[41] W.-J. Li and H.-B. Zhang, Holographic non-relativistic fermionic fixed point and bulk dipole coupling, JHEP 11 (2011) 018 [arXiv:1110.4559] [INSPIRE].

[42] J.-P. Wu, The analytical treatments on the low energy behaviors of the holographic non-relativistic fermions, Phys. Lett. B 723 (2013) 448 [INSPIRE]. 